\section{Adaptive Stabilization of a Class of Nonlinear Systems With Nonparametric Uncertainty}

\author{
Alexander V. Roup and Dennis S. Bernstein
}

\begin{abstract}
We consider adaptive stabilization for a class of nonlinear second-order systems. Interpreting the system states as position and velocity, the system is assumed to have unknown, nonparametric position-dependent damping and stiffness coefficients. Lyapunov methods are used to prove global convergence of the adaptive controller. Furthermore, the controller is shown to be able reject constant disturbances and to asymptotically track constant commands. For illustration, the controller is used to stabilize the van der Pol limit cycle, the Duffing oscillator with multiple equilibria, and several other example systems.
\end{abstract}

Index Terms-Adaptive stabilization, nonlinear systems, nonparametric uncertainty.

\section{INTRODUCTION}

There are many applications of control in which a reliable model of the dynamical system is not available. This can occur if the system is not amenable to analytical modeling due to unknown or unpredictably changing physics, or if identification is not feasible due to instability, disturbances, sensor noise, poor repeatability, or high cost. Under high levels of uncertainty, robust control may be ineffective and adaptive control is warranted.

For implementation, adaptive controllers generally require some knowledge about the plant in the form of parameter or transfer function estimates, and this knowledge may be available prior to operation due to analytical modeling or off-line identification, or it may be determined during operation through concurrent identification. The former case is usually termed direct adaptive control, while the latter constitutes indirect adaptive control. In addition, adaptive control methods often depend on structural assumptions about the plant, for example, passivity and relative degree.

In this note we consider the problem of adaptive stabilization and constant disturbance rejection for a class of second-order nonlinear systems under full-state feedback. In Section II, we present the adaptive controller and prove convergence of the plant states. The novel aspect of this controller is the fact that global convergence is guaranteed under nonparametric assumptions about the nonlinearities. Interpreting the system states as position and velocity, the system is assumed to have unknown, position-dependent damping and stiffness coefficients, which are assumed only to be continuous and lower bounded. Furthermore, these lower bounds need not be known. A classical system satisfying these assumptions is the van der Pol oscillator whose limit cycle is stabilized by our controller without knowledge about the form of the position-dependent, sign-varying damping.

The form of our controller is similar to direct adaptive controllers developed for linear systems. Related theory can be found in [1]-[6], where the emphasis is on model following control. For adaptive stabilization, a self-contained treatment of the relevant ideas and techniques is given in [7], where the stability of the closed-loop system is proven for linear plants and the controller is applied to nonlinear plants. In [8],

Manuscript received November 22, 1999; revised December 18, 2000 and April 20, 2001. Recommended by Associate Editor P. Tomei. This work was supported in part by the Air Force Office of Scientific Research under Grant F49620-98-1-0037 and the François-Xavier Bagnoud Foundation Fellowship.

The authors are with the Department of Aerospace Engineering, the University of Michigan, Ann Arbor, MI 48109-2140 USA (e-mail: aroup@umich.edu; dsbaero@umich.edu).

Publisher Item Identifier S 0018-9286(01)10360-0. the controller presented in [7] is applied to motion control experiments. The main difference between the controller of this note and [7] is a condition on the sign of the $(1,2)$ entry of the Lyapunov matrix $P$. In [7] this sign condition is implicit in the solution of the Lyapunov equation for second-order systems in companion form. Numerical experiments show that violation of this condition can destabilize the closed-loop system.

Since we assume full-state feedback control in companion coordinates, that is, position and velocity measurements, our controller is a direct adaptive controller, and thus parameter estimates are not needed. In addition, full-state feedback availability avoids the need for positivity assumptions. Extensions to output feedback, nonconstant disturbance rejection, and model reference adaptive control will be considered in future work.

\section{ADAPTIVE STABILIZATION}

We wish to determine a feedback control law for the nonlinear system

$$
m \ddot{q}(t)+g(q(t)) \dot{q}(t)+f(q(t)) q(t)=b u(t)+d
$$

where $f: \mathbf{R} \rightarrow \mathbf{R}, g: \mathbf{R} \rightarrow \mathbf{R}$, and $m, b, d \in \mathbf{R}$, such that $q(t) \rightarrow 0$ and $\dot{q}(t) \rightarrow 0$ as $t \rightarrow \infty$. We assume that (1) is uncertain in the following sense. The functions $f$ and $g$ are known to be locally Lipschitz on $\mathbf{R}$ and lower bounded but are otherwise uncertain, the constant $m$ is known to be positive but is otherwise uncertain, the constant $b$ is known to be nonzero with known sign but is otherwise uncertain, and the constant $d$ is uncertain.

Under the above assumptions, the control law

$$
u(t)=k_{1}(t) q(t)+k_{2}(t) \dot{q}(t)+\phi(t)
$$

where the gains $k_{1}(t), k_{2}(t)$ and the parameter $\phi(t)$ are adapted, will be used to obtain $q(t) \rightarrow 0$ and $\dot{q}(t) \rightarrow 0$ as $t \rightarrow \infty$. Note that if $u \equiv 0$ and $d=0$, then $(q, \dot{q})=(0,0)$ is an equilibrium of (1) but not necessarily the only equilibrium. Furthermore, if $u \equiv 0$ but $d \neq 0$, then $(q, \dot{q})=(0,0)$ is not an equilibrium of (1).

Define the state

$$
x(t)=\left[\begin{array}{l}
x_{1}(t) \\
x_{2}(t)
\end{array}\right] \triangleq\left[\begin{array}{l}
q(t) \\
\dot{q}(t)
\end{array}\right]
$$

and the gain matrix

$$
K(t) \triangleq\left[k_{1}(t) \quad k_{2}(t)\right]
$$

Dynamic variables will henceforth be written without a time dependence argument. The state equation for (1), (2) is

$$
\dot{x}=\left[\begin{array}{c}
x_{2} \\
(1 / m)\left[b K x+b \phi+d-x_{2} g\left(x_{1}\right)-x_{1} f\left(x_{1}\right)\right.
\end{array}\right] .
$$

Let $P \triangleq\left[\begin{array}{cc}p_{1} & p_{12} \\ p_{12} & p_{2}\end{array}\right]$ be positive-definite, with $p_{12}>0$. Let

$$
\alpha \triangleq \inf _{q \in \mathbf{R}} f(q) \quad \beta \triangleq \inf _{q \in \mathbf{R}} g(q)
$$

and define the set

$$
\begin{aligned}
\mathcal{K}_{s}=\left\{\left[\begin{array}{ll}
k_{1 s} & k_{2 s}
\end{array}\right]: b k_{1 s}<\alpha\right. \\
\left.b k_{2 s}<\beta-\frac{m}{p_{2}}\left(\frac{m p_{1}^{2}}{4 p_{12}\left(\alpha-b k_{1 s}\right)}+p_{12}\right)\right\} .
\end{aligned}
$$


Lemma 1: $\mathcal{K}_{s}$ is not empty. Now, let $K_{s}=\left[\begin{array}{ll}k_{1 s} & k_{2 s}\end{array}\right] \in \mathcal{K}_{s}$ and define $R \in \mathbf{R}^{2 \times 2}, \tilde{f}: \mathbf{R} \rightarrow \mathbf{R}$, and $\tilde{g}: \mathbf{R} \rightarrow \mathbf{R}$ by

$$
\begin{gathered}
R \triangleq\left[\begin{array}{cc}
\frac{p_{12}}{m}\left(\alpha-b k_{1 s}\right) & -\frac{1}{2} p_{1} \\
-\frac{1}{2} p_{1} & \frac{p_{2}}{m}\left(\beta-b k_{2 s}\right)-p_{12}
\end{array}\right], \\
\tilde{f}(q) \triangleq f(q)-b k_{1 s}, \quad \tilde{g}(q) \triangleq g(q)-b k_{2 s} .
\end{gathered}
$$

Then $R$ is positive-definite, and $\tilde{f}(q)>0$ and $\tilde{g}(q)>0$ for all $q \in \mathbf{R}$. Furthermore, with $K=K_{s}$ and $\phi=-d / b$, the origin of (5) is a globally asymptotically stable equilibrium.

Proof: The first inequality of (7) is an upper bound on $b k_{1 s}$. The second inequality of (7) is an upper bound on $b k_{2 s}$ in terms of $b k_{1 s}$. Since $b k_{1 s}$ and $b k_{2 s}$ are only bounded above, $\mathcal{K}_{s}$ is not empty.

The matrix $R$ is positive-definite if and only if

$$
\begin{aligned}
& \frac{p_{12}}{m}\left(\alpha-b k_{1 s}\right)>0, \\
& \frac{p_{2}}{m}\left(\beta-b k_{2 s}\right)-p_{12}>0, \\
& \frac{p_{12}}{m}\left(\alpha-b k_{1 s}\right)\left[\frac{p_{2}}{m}\left(\beta-b k_{2 s}\right)-p_{12}\right]>\frac{1}{4} p_{1}^{2} .
\end{aligned}
$$

The first inequality of (7) implies (10), while the second inequality of (7) implies (12). Furthermore, (10) and (12) imply (11). Therefore, since $K_{s} \in \mathcal{K}_{s}, R$ is positive-definite.

To show that $\tilde{f}(q)>0$ and $\tilde{g}(q)>0$ for all $q \in \mathbf{R}$, note that for all $q \in \mathbf{R}$

$$
\tilde{f}(q)=f(q)-b k_{1 s} \geq \alpha-b k_{1 s}>0,
$$

and

$$
\begin{aligned}
\tilde{g}(q) & =g(q)-b k_{2 s} \geq \beta-b k_{1 s} \\
& >\frac{m}{p_{2}}\left(\frac{m p_{1}^{2}}{4 p_{12}\left(\alpha-b k_{1 s}\right)}+p_{12}\right)>0 .
\end{aligned}
$$

Next we show that if $K=K_{s} \in \mathcal{K}_{s}$ and $\phi=-d / b$, then the origin of (5) is a globally asymptotically stable equilibrium. The closed-loop system (5) can be written in the form

$$
\dot{x}=\left[\begin{array}{c}
x_{2} \\
(1 / m)\left[-x_{2} \tilde{g}\left(x_{1}\right)-x_{1} \tilde{f}\left(x_{1}\right)\right]
\end{array}\right] .
$$

Note that $x=0$ is the unique equilibrium of (15) since $\tilde{f}(q)>0$ and $\tilde{g}(q)>0$ for all $q \in \mathbf{R}$. Consider the Lyapunov candidate for (5) given by

$$
V(x)=\frac{1}{2} x^{T} P x+\frac{p_{2}}{m} \int_{0}^{x_{1}} \sigma \tilde{f}(\sigma) d \sigma+\frac{p_{12}}{m} \int_{0}^{x_{1}} \eta \tilde{g}(\eta) d \eta
$$

which is positive-definite and radially unbounded. The derivative of $V$ along the system trajectory is

$$
\begin{aligned}
\dot{V}(x)= & x^{T} P \dot{x}+\frac{p_{2}}{m} x_{1} \tilde{f}\left(x_{1}\right) \dot{x}_{1}+\frac{p_{12}}{m} x_{1} \tilde{g}\left(x_{1}\right) \dot{x}_{1} \\
= & -\frac{p_{12}}{m} \tilde{f}\left(x_{1}\right) x_{1}^{2}+p_{1} x_{1} x_{2}-\left(\frac{p_{2}}{m} \tilde{g}\left(x_{1}\right)-p_{12}\right) x_{2}^{2} \\
\leq & -\frac{p_{12}}{m}\left(\alpha-b k_{1 s}\right) x_{1}^{2}+p_{1} x_{1} x_{2} \\
& -\left(\frac{p_{2}}{m}\left(\beta-b k_{2 s}\right)-p_{12}\right) x_{2}^{2} \\
= & -x^{T} R x .
\end{aligned}
$$

Since $R$ is positive definite, the origin of (5) is globally asymptotically stable.

Now, consider the system (5) with the adaptation law

$$
\begin{aligned}
\dot{x} & =\left[\begin{array}{c}
x_{2} \\
(1 / m)\left[b K x+b \phi+d-x_{2} g\left(x_{1}\right)-x_{1} f\left(x_{1}\right)\right]
\end{array}\right] \\
\dot{K} & =-\Gamma B_{0}^{T} P x x^{T} \Lambda \\
\dot{\phi} & =-B_{0}^{T} P x \lambda
\end{aligned}
$$

where $\Gamma \in \mathbf{R}, P \in \mathbf{R}^{2 \times 2}, \Lambda \in \mathbf{R}^{2 \times 2}$, and $\lambda \in \mathbf{R}$. Let $\Gamma>0, \Lambda$ be positive-definite, $\lambda>0$, and define $B_{0} \triangleq[0 \operatorname{sign}(b)]^{T}$.

The equilibria set of the closed-loop system (18)-(20) is

$\mathcal{E}=\left\{(x, K, \phi) \in \mathbf{R}^{2} \times \mathbf{R}^{1 \times 2} \times \mathbf{R}: x=\left[\begin{array}{ll}0 & 0\end{array}\right]^{T}\right.$,

$$
\left.K \in \mathbf{R}^{1 \times 2}, \phi=-d / b\right\} \text {. }
$$

Define the subset of equilibria

$$
\mathcal{E}_{s}=\left\{\left(x, K_{s}, \phi\right) \in \mathcal{E}: K_{s} \in \mathcal{K}_{s}\right\} .
$$

Theorem 1: Every element of $\mathcal{E}_{s}$ is a Lyapunov stable equilibrium of the closed-loop system (18)-(20). Furthermore, the functions $x, K$, and $\phi$ satisfying (18)-(20) are bounded, and $x(t) \rightarrow\left[\begin{array}{ll}0 & 0\end{array}\right]^{T}$ and $\phi(t) \rightarrow$ $-d / b$ as $t \rightarrow \infty$. Define

Proof: Let $\left(\left[\begin{array}{ll}0 & 0\end{array}\right]^{T}, K_{s},-d / b\right) \in \mathcal{E}_{s}$, where $K_{s}=\left[\begin{array}{ll}k_{1 s} & k_{2 s}\end{array}\right]$.

$$
\begin{aligned}
& \tilde{k}_{1} \triangleq k_{1}-k_{1 s}, \quad \tilde{k}_{2} \triangleq k_{2}-k_{2 s}, \quad \tilde{K} \triangleq K-K_{s}, \\
& \tilde{\phi} \triangleq \phi+d / b,
\end{aligned}
$$

the matrix $R$ as in (8), and the functions $\tilde{f}$ and $\tilde{g}$ as in (9). Note that since $\left[k_{1 s} k_{2 s}\right] \in \mathcal{K}_{s}$ it follows from Lemma 1 that $R$ is positive-definite and $\tilde{f}(q)>0$ and $\tilde{g}(q)>0$ for all $q \in \mathbf{R}$.

The closed-loop system (18)-(20) can be written in the form

$$
\begin{aligned}
& \dot{x}=\left[\begin{array}{c}
x_{2} \\
(1 / m)\left[b \tilde{K} x+b \tilde{\phi}-x_{2} \tilde{g}\left(x_{1}\right)-x_{1} \tilde{f}\left(x_{1}\right)\right]
\end{array}\right], \\
& \dot{\tilde{K}}=-\Gamma B_{0}^{T} P x x^{T} \Lambda \text {, } \\
& \dot{\tilde{\phi}}=-B_{0}^{T} P x \lambda \text {. }
\end{aligned}
$$

The Lyapunov analysis that follows concerns the stability of the equilibrium point $(x, \tilde{K}, \tilde{\phi})=\left(\left[\begin{array}{ll}0 & 0\end{array}\right]^{T},\left[\begin{array}{ll}0 & 0\end{array}\right], 0\right)$ of (24)-(26). Note that the equilibrium point $(x, \tilde{K}, \tilde{\phi})=\left(\left[\begin{array}{ll}0 & 0\end{array}\right]^{T},\left[\begin{array}{ll}0 & 0\end{array}\right], 0\right)$ of $(24)-(26)$ corresponds to the equilibrium point $(x, K, \phi)=\left(\left[\begin{array}{ll}0 & 0\end{array}\right]^{T}, K_{s},-d / b\right)$ of (18)-(20) through the coordinate transformation given by (23).

Consider the Lyapunov candidate for the system (24)-(26) given by

$$
\begin{array}{rl}
V(x, \tilde{K}, \tilde{\phi})=\frac{1}{2} x^{T} & P x+\frac{p_{2}}{m} \int_{0}^{x_{1}} \sigma \tilde{f}(\sigma) d \sigma+\frac{p_{12}}{m} \int_{0}^{x_{1}} \eta \tilde{g}(\eta) d \eta \\
+ & \frac{|b|}{2 m} \operatorname{tr} \Gamma^{-1} \tilde{K} \Lambda^{-1} \tilde{K}^{T}+\frac{|b|}{2 m} \operatorname{tr} \tilde{\phi} \lambda^{-1} \tilde{\phi}^{T}
\end{array}
$$

which is positive-definite and radially unbounded. The derivative of $V$ along the system trajectory is

$$
\begin{aligned}
& \dot{V}(x, \tilde{K}, \tilde{\phi})= x^{T} P \dot{x}+\frac{p_{2}}{m} x_{1} \tilde{f}\left(x_{1}\right) \dot{x}_{1}+\frac{p_{12}}{m} x_{1} \tilde{g}\left(x_{1}\right) \dot{x}_{1} \\
&+\frac{|b|}{m} \operatorname{tr} \Gamma^{-1} \tilde{K} \Lambda^{-1} \dot{\tilde{K}}^{T}+\frac{|b|}{m} \operatorname{tr} \tilde{\phi} \lambda^{-1} \dot{\tilde{\phi}}^{T} \\
&=\left(x_{1} p_{1}+x_{2} p_{12}\right) x_{2}+\frac{1}{m}\left(x_{1} p_{12}+x_{2} p_{2}\right) \\
& \times\left(b \tilde{K} x+b \tilde{\phi}-x_{2} \tilde{g}\left(x_{1}\right)-x_{1} \tilde{f}\left(x_{1}\right)\right) \\
&+\frac{p_{2}}{m} x_{1} x_{2} \tilde{f}\left(x_{1}\right)+\frac{p_{12}}{m} x_{1} x_{2} \tilde{g}\left(x_{1}\right) \\
&+\frac{|b|}{m} \operatorname{tr} \Gamma^{-1} \tilde{K} \Lambda^{-1} \dot{\tilde{K}}^{T}+\frac{|b|}{m} \operatorname{tr} \tilde{\phi} \lambda^{-1} \dot{\tilde{\phi}}^{T} \\
&=\left(x_{1} p_{1}+x_{2} p_{12}\right) x_{2}-\frac{1}{m}\left(x_{1} p_{12}+x_{2} p_{2}\right) \\
& \times\left(\tilde{g}\left(x_{1}\right) x_{2}+x_{1} \tilde{f}\left(x_{1}\right)\right)+\frac{p_{2}}{m} x_{1} x_{2} \tilde{f}\left(x_{1}\right) \\
&+\frac{p_{12}}{m} x_{1} x_{2} \tilde{g}\left(x_{1}\right)+\frac{|b|}{m} \operatorname{tr} \tilde{K}\left(x x^{T} P B_{0}\right. \\
&+\Lambda-1 \\
&\left.\dot{\tilde{K}}^{T} \Gamma^{-1}\right)+\frac{|b|}{m} \operatorname{tr} \tilde{\phi}\left(x^{T} P B_{0}+\lambda^{-1} \dot{\tilde{\phi}}^{T}\right) \\
&=-\frac{p_{12}}{m} \tilde{f}\left(x_{1}\right) x_{1}^{2}+p_{1} x_{1} x_{2} \\
&-\left(\frac{p_{2}}{m} \tilde{g}\left(x_{1}\right)-p_{12}\right) x_{2}^{2}
\end{aligned}
$$




$$
\begin{aligned}
\leq & -\frac{p_{12}}{m}\left(\alpha-b k_{1 s}\right) x_{1}^{2}+p_{1} x_{1} x_{2} \\
& -\left(\frac{p_{2}}{m}\left(\beta-b k_{2 s}\right)-p_{12}\right) x_{2}^{2} \\
= & -x^{T} R x \\
\leq & 0
\end{aligned}
$$

for all $(x, \tilde{K}, \tilde{\phi}) \in \mathbf{R}^{2} \times \mathbf{R}^{1 \times 2} \times \mathbf{R}$. Therefore, $\left(\left[\begin{array}{ll}0 & 0\end{array}\right]^{T}, K_{s},-d / b\right)$ is a Lyapunov stable equilibrium of (18)-(20). Since $V(x, \tilde{K}, \tilde{\phi})>0$ and $\dot{V}(x, \tilde{K}, \tilde{\phi}) \leq 0$ it follows that $V(x, \tilde{K}, \tilde{\phi})$ is bounded. Since, in addition, $V(x, \tilde{K}, \tilde{\phi})$ is radially unbounded, it follows that $x, K$, and $\phi$ are bounded.

Next, to prove $x(t) \rightarrow\left[\begin{array}{ll}0 & 0\end{array}\right]^{T}$ and $\phi(t) \rightarrow-d / b$ as $t \rightarrow \infty$, assume $(x(0), \tilde{K}(0), \tilde{\phi}(0)) \neq\left(\left[\begin{array}{ll}0 & 0\end{array}\right]^{T},\left[\begin{array}{ll}0 & 0\end{array}\right], 0\right)$ and let $\rho \triangleq V(x(0), \tilde{K}(0), \tilde{\phi}(0))>0$ and

$$
\mathcal{N} \triangleq\{(x, \tilde{K}, \tilde{\phi}): \dot{V}(x, \tilde{K}, \tilde{\phi})=0, V(x, \tilde{K}, \tilde{\phi}) \leq \rho\} .
$$

Note that because $R$ is positive-definite, $\dot{V}(x, \tilde{K}, \tilde{\phi})=0$ implies that $x=\left[\begin{array}{ll}0 & 0\end{array}\right]^{T}$. Conversely, substituting $x=\left[\begin{array}{ll}0 & 0\end{array}\right]^{T}$ into (28) gives $\dot{V}(x, \tilde{K}, \tilde{\phi})=0$. Therefore $\dot{V}(x, \tilde{K}, \tilde{\phi})=0$ if and only if $x=\left[\begin{array}{ll}0 & 0\end{array}\right]^{T}$. Hence

$$
\mathcal{N}=\left\{(x, \tilde{K}, \tilde{\phi}): x=\left[\begin{array}{ll}
0 & 0
\end{array}\right]^{T}, V(x, \tilde{K}, \tilde{\phi}) \leq \rho\right\} .
$$

Substituting $x \equiv\left[\begin{array}{ll}0 & 0\end{array}\right]^{T}$ into (24)-(26) it can be seen that $\dot{x}=\left[\begin{array}{ll}0 & 0\end{array}\right]^{T}$ if and only if $\tilde{\phi}=0$. It follows that the largest invariant subset of $\mathcal{N}$ is given by

$$
\mathcal{M}=\{(x, \tilde{K}, \tilde{\phi}) \in \mathcal{N}: \tilde{\phi}=0\} .
$$

Now, LaSalle's theorem ([9, Th. 3.4]) implies that $(x, \tilde{K}, \tilde{\phi}) \rightarrow \mathcal{M}$ as $t \rightarrow \infty$. It follows that $x(t) \rightarrow\left[\begin{array}{ll}0 & 0\end{array}\right]^{T}$ and $\phi(t) \rightarrow-d / b$ as $t \rightarrow \infty$.

Note that the lower bounds $\alpha$ and $\beta$ for $f$ and $g$ defined by (6) are used only in the proof and need not be known to implement the adaptive controller (2), (19), (20).

For the case in which (1) is linear, Theorem 1 specializes to [7, Cor. 3.1]. In [7], the matrix $P$ was obtained as the solution to the Lyapunov equation $0=A_{s}^{T} P+P A_{s}+R$, where $A_{s}=A+B K_{s}$ and $R$ is an arbitrary positive definite matrix. It can be seen that when $A_{s}$ is in canonical form, the $(1,2)$ entry of $P$ is always positive. Hence the requirement $p_{12}>0$ represents no loss of generality when Theorem 1 is applied to linear plants.

The adaptive controller (2), (19), (20) can be used to asymptotically track constant position references. Define the position error

$$
e(t) \triangleq q(t)-r
$$

where $r$ is a constant reference. In terms of $e$ and $r$, the system equation (1) has the form

$$
\begin{aligned}
m \ddot{e}(t)+g(e(t)+r) \dot{e}(t)+f(e(t)+r)(e(t)+r) & \\
& =b u(t)+d .
\end{aligned}
$$

Now defining

$$
\begin{aligned}
f_{1}(e) & \triangleq \frac{1}{e}[f(e+r)(e+r)-f(r) r], \\
g_{1}(e) & \triangleq g(e+r) \\
d_{1} & \triangleq d-f(r) r
\end{aligned}
$$

(34) can be written as

$$
m \ddot{e}(t)+g_{1}(e(t)) \dot{e}(t)+f_{1}(e(t)) e(t)=b u(t)+d_{1}
$$

which is identical in form to (1). The adaptive controller (2), (19), (20) can be applied to (38) using the state definition $x=\left[\begin{array}{ll}e & \dot{q}\end{array}\right]^{T}$ to give $q \rightarrow r$ and $\dot{q} \rightarrow 0$ as $t \rightarrow \infty$.

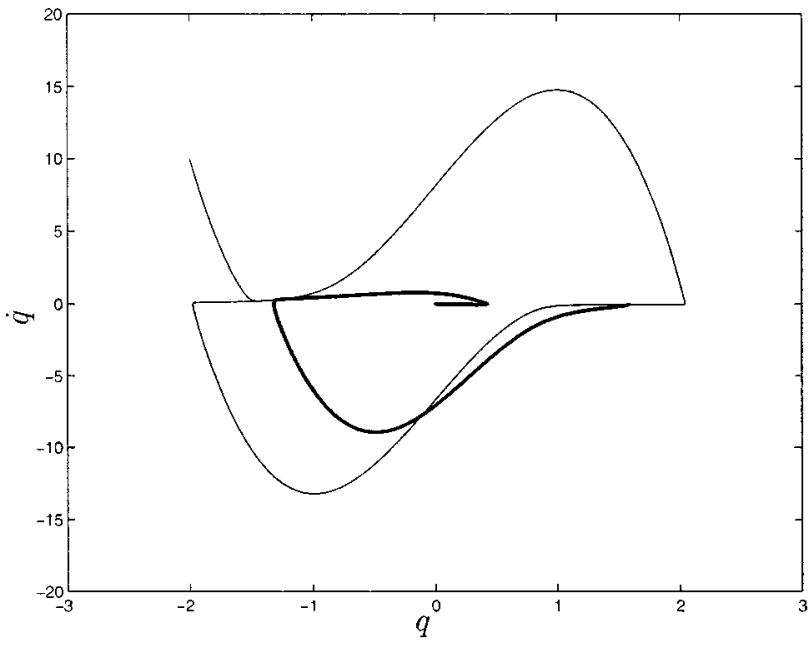

Fig. 1. System trajectory in the $q, \dot{q}$ plane for Example 1, the van der Pol oscillator. Initial conditions are $q=-1, \dot{q}=1, k_{1}=0, k_{2}=0$, and $\phi=0$. Uncontrolled response is shown with thin line. Controlled response is shown with thick line.

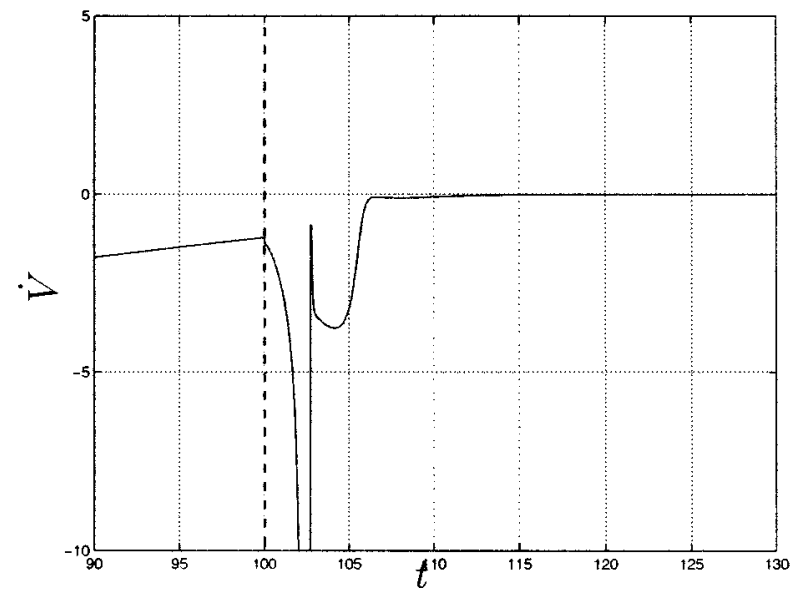

Fig. 2. Time history of $\dot{V}$ for Example 1, the van der Pol oscillator. Control system is activated at $t=100$, which is marked with a vertical dashed line.

\section{NUMERICAL EXAMPLES}

Example 1: Consider the van der Pol oscillator with constant disturbance given by

$$
\ddot{q}+10\left(q^{2}-1\right) \dot{q}+q=u+0.9 .
$$

For this system, $g(q)=10\left(q^{2}-1\right)$ and $f(q)=1$, which are both bounded from below. Note that with the constant disturbance term $d=0.9$, the open-loop system does not have an equilibrium point at $(q, \dot{q})=(0,0)$. However, the closed-loop system (39), (2), (19), (20) has the equilibria set $\left.\mathcal{E}=\left\{\left(\left[\begin{array}{ll}q & \dot{q}\end{array}\right]^{T}, K, \phi\right): q=0, \dot{q}=0, K \in \mathrm{R}^{1 \times 2}, \phi=-0.9\right)\right\}$.

Choose

$$
P=\left[\begin{array}{cc}
1 & 0.5 \\
0.5 & 1
\end{array}\right], \quad \Gamma=1, \quad \Lambda=\left[\begin{array}{ll}
1 & 0 \\
0 & 1
\end{array}\right], \quad \lambda=1 .
$$

Fig. 1 shows the system trajectory in the $q, \dot{q}$ plane. The uncontrolled system is allowed to approach a limit cycle, and then the adaptive control system is activated at $t=100 . V(x, \tilde{k}, \tilde{\phi})$ was calculated using (27) with the parameters $k_{1 s}=0$ and $k_{2 s}=-15$ selected to satisfy (7). Fig. 2 shows the time history of $\dot{V}$, and Fig. 3 shows the time history of $k_{1}, k_{2}$, and $\phi$ before and after control system activation. The states $q$ and $\dot{q}$ of the closed-loop system converge to $q=0$ and $\dot{q}=0$. 


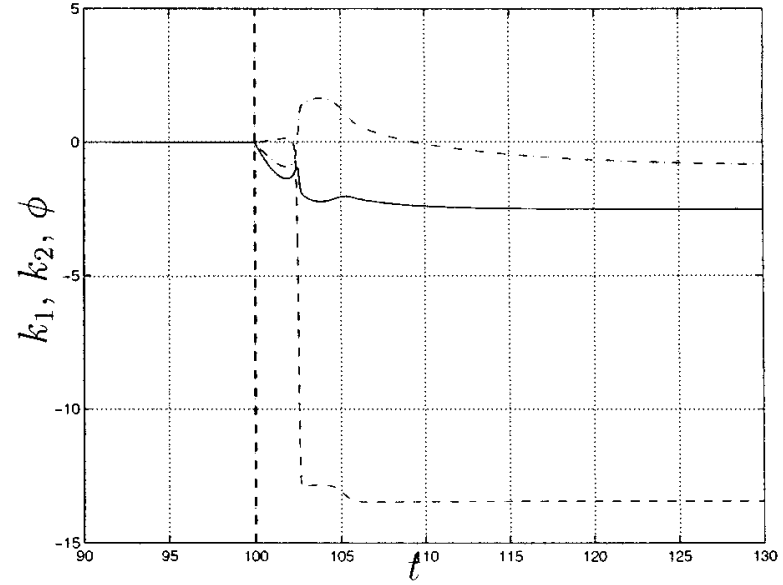

Fig. 3. Time history of $k_{1}(-), k_{2}(-)$, and $\phi(-$.$) for Example 1, the van der$ Pol oscillator. Control system is activated at $t=100$, which is marked with a vertical dashed line.

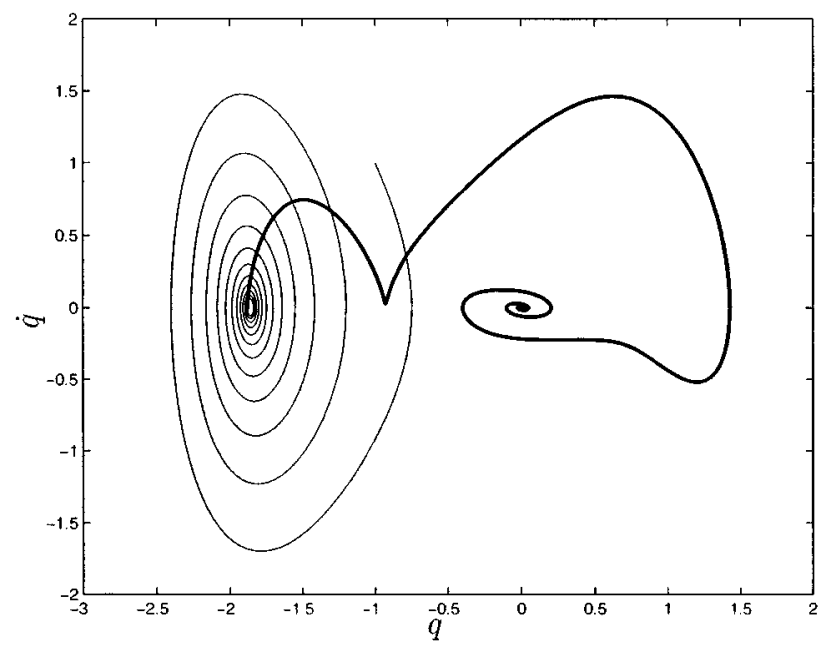

Fig. 4. System trajectory in the $q, \dot{q}$ plane for Example 2, the Duffing oscillator. Initial conditions are $q=-1, \dot{q}=1, k_{1}=0, k_{2}=0$, and $\phi=0$. Uncontrolled response is shown with thin line. Controlled response is shown with thick line.

Example 2: Next, consider the Duffing oscillator with constant disturbance given by

$$
\ddot{q}+(1 / 4) \dot{q}+\left(q^{2}-4\right) q=u+1 .
$$

The uncontrolled system has stable foci at $(-1.86,0)$ and $(2.11,0)$ and a saddle at $(-0.25,0)$. For this system, $g(q)=1 / 4$ and $f(q)=q^{2}-4$, which are bounded from below.

Choose controller parameters as in (40). Fig. 4 shows the system trajectory in the $q, \dot{q}$ plane. The uncontrolled system is allowed to approach a stable focus, and the adaptive control system is activated at $t=30$. The states $q$ and $\dot{q}$ of the closed-loop system converge to $q=0$ and $\dot{q}=0$.

Example 3: Next consider the nonlinear system with randomly generated piecewise linear stiffness and damping given by (1) with $m=$ $1, b=1$, and $d=0$. The stiffness and damping functions $f$ and $g$ are the randomly generated piecewise linear functions shown in Fig. 5. These nonlinear functions can be viewed as interpolations of lookup table data.

Choose controller parameters as in (40). Fig. 6 shows the system trajectory in the $q, \dot{q}$ plane. The uncontrolled system is allowed to diverge,

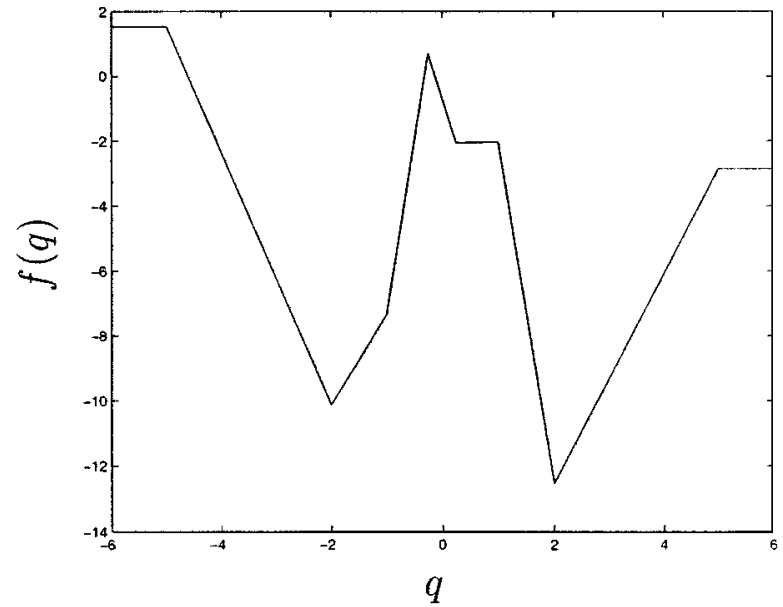

(a)

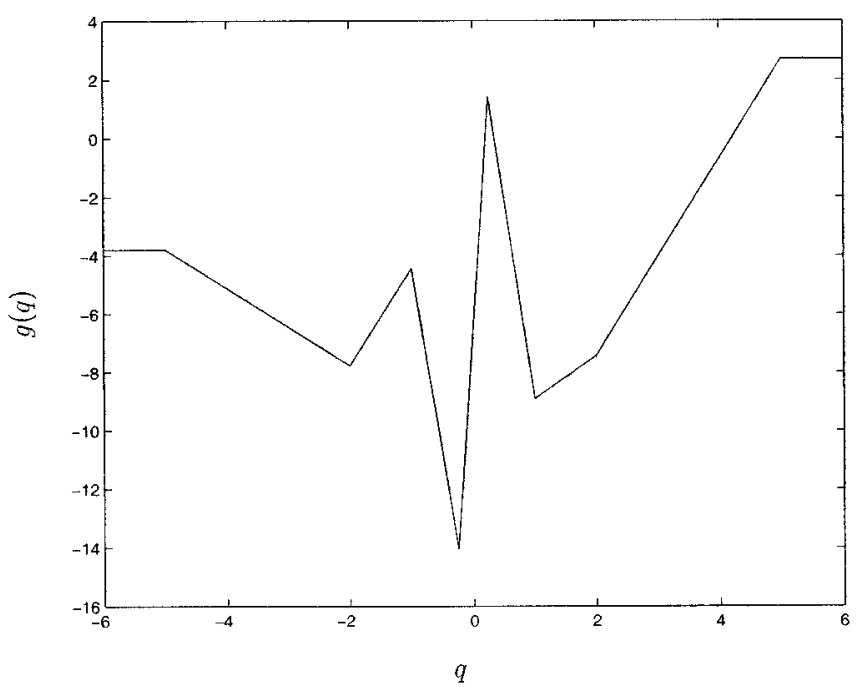

(b)

Fig. 5. Randomly generated piecewise linear functions $f$ and $g$ used in Example 3.

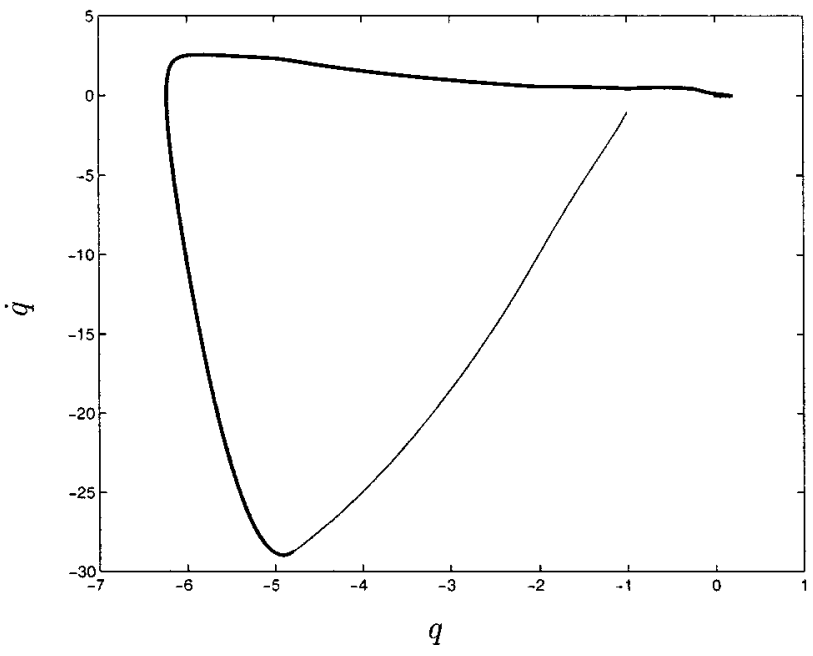

Fig. 6. System trajectory in the $q, \dot{q}$ plane for Example 3. Initial conditions are $q=-1, \dot{q}=-1, k_{1}=0, k_{2}=0$, and $\phi=0$. Uncontrolled response is shown with thin line. Controlled response is shown with thick line.

and the adaptive control system is activated at $t=0.4$. The states $q$ and $\dot{q}$ of the closed-loop system converge to $q=0$ and $\dot{q}=0$. 


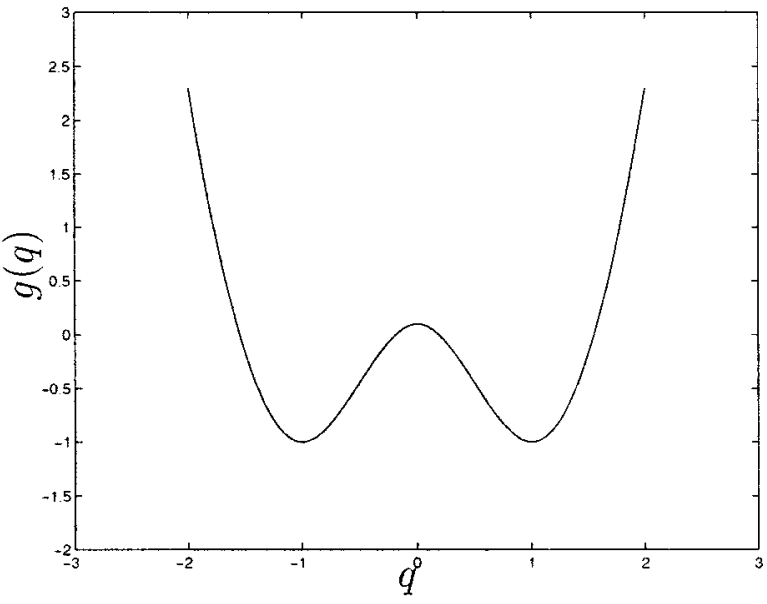

Fig. 7. Function $g$ for Example 4.

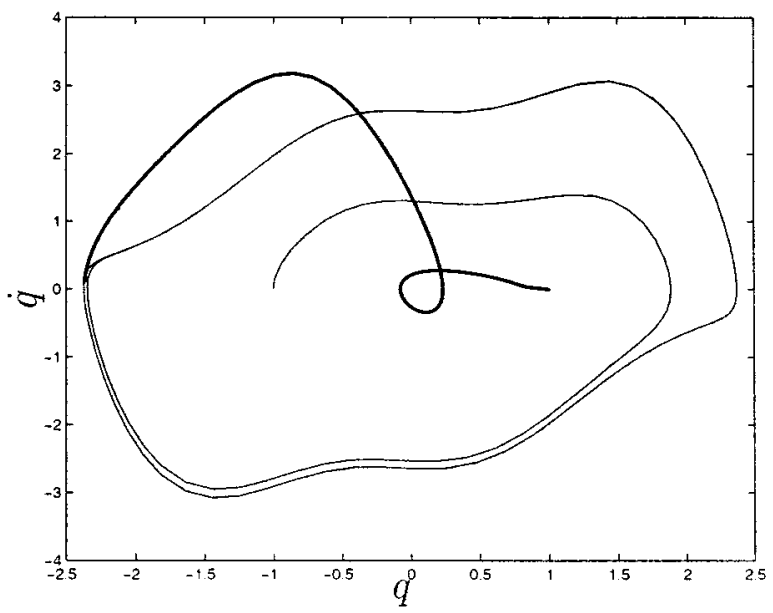

Fig. 8. System trajectory in the $q, \dot{q}$ plane for Example 4. Initial conditions are $q=-1, \dot{q}=0, k_{1}=0, k_{2}=0$, and $\phi=0$. Uncontrolled response is shown with thin line. Controlled response is shown with thick line.

Example 4: Consider the nonlinear system given by

$$
\ddot{q}+g(q) \dot{q}+q=u
$$

where

$$
g(q)= \begin{cases}2.2\left|q^{3}\right|-3.3 q^{2}+0.1, & |q|<1 \\ 3.3(|q|-1)^{2}-1, & |q| \geq 1\end{cases}
$$

The damping function $g$ is shown in Fig. 7. We wish to drive the state $q$ to $q=1$. With the reference input $r=1$, choose controller parameters as in (40) and use the modified control

$$
\begin{aligned}
u & =k_{1}(q-r)+k_{2} \dot{q}+\phi \\
\dot{K} & =-\Gamma B_{0}^{T} P\left[\begin{array}{c}
q-r \\
\dot{q}
\end{array}\right][q-r \dot{q}] \Lambda \\
\dot{\phi} & =-B_{0}^{T} P\left[\begin{array}{c}
q-r \\
\dot{q}
\end{array}\right] \lambda .
\end{aligned}
$$

Note that with $r \equiv 0$, (44)-(46) are identical to (2), (19), (20).

Choose controller parameters as in (40). Fig. 8 shows the system trajectory in the $q, \dot{q}$ plane. Beginning from the initial condition $q=$

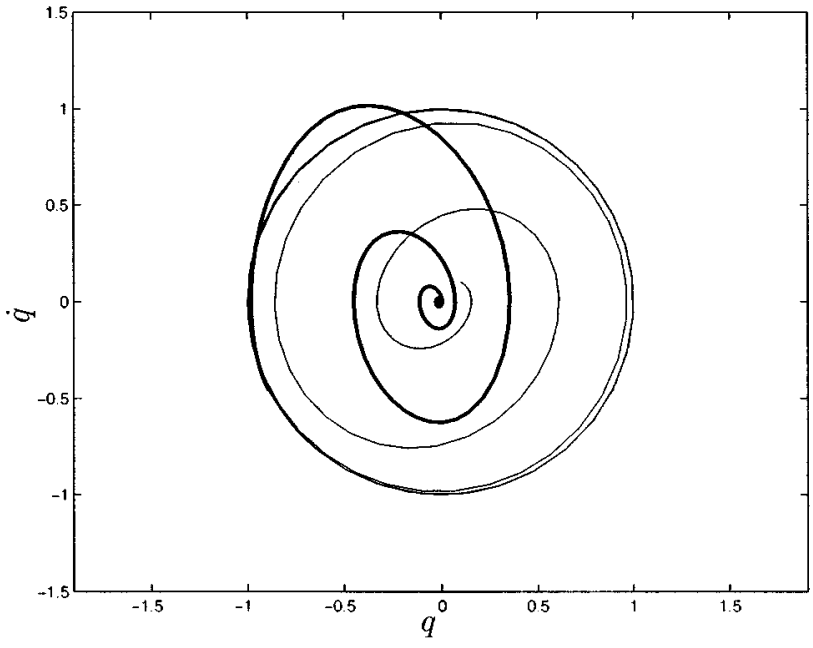

Fig. 9. System trajectory in the $q, \dot{q}$ plane for Example 5. Initial conditions are $q=0.1, \dot{q}=0.1, k_{1}=0, k_{2}=0$, and $\phi=0$. Uncontrolled response is shown with thin line. Controlled response is shown with thick line.

-1 and $\dot{q}=0$, the uncontrolled system approaches a limit cycle. The adaptive control system is activated at $t=19$. The states $q$ and $\dot{q}$ of the closed-loop system converge to $q=1$ and $\dot{q}=0$.

Example 5: Consider the nonlinear system given by

$$
\ddot{q}+\lambda\left(q^{2}+\omega^{-2} \dot{q}^{2}-a^{2}\right) \dot{q}+\omega^{2} q=u .
$$

Uncontrolled trajectories of (47) with nonzero initial conditions approach a sinusoidal limit cycle with amplitude $a$ and frequency $\omega$. The parameter $\lambda$ adjusts the rate of convergence to the limit cycle. Theorem 1 does not apply to this example because $g$ is a function of $\dot{q}$ as well as $q$. For this example choose parameters $a=1, \omega=1$, and $\lambda=0.5$.

Choose controller parameters as in (40). Figure 9 shows the system trajectory in the $q, \dot{q}$ plane. Beginning from the initial condition $q=0.1$ and $\dot{q}=0.1$, the uncontrolled system approaches a limit cycle. Then the adaptive control system is activated at $t=35$. The state $q$ and $\dot{q}$ of the closed-loop system converge to $q=0$ and $\dot{q}=0$.

\section{REFERENCES}

[1] K. S. Narendra and A. M. Annaswamy, Stable Adaptive Systems. Upper Saddle River, NJ: Prentice-Hall, 1989.

[2] S. Sastry and M. Bodson, Adaptive Control: Stability, Convergence, and Robustness. Upper Saddle River, NJ: Prentice-Hall, 1989.

[3] K. J. Åström and B. Wittenmark, Adaptive Control, 2nd ed. Reading, MA: Addison-Wesley, 1995.

[4] M. Krstic, I. Kanellakopoulos, and P. Kokotovic, Nonlinear and Adaptive Control Design. New York, NY: Wiley, 1995.

[5] P. A. Ioannou and J. Sun, Robust Adaptive Control. Upper Saddle River, NJ: Prentice-Hall, 1996.

[6] H. Kaufman, I. Barkana, and K. Sobel, Direct Adaptive Control Algorithms: Theory and Applications, 2nd ed. New York: Springer-Verlag, 1998.

[7] J. Hong and D. S. Bernstein, "Adaptive stabilization of nonlinear oscillators using direct adaptive control," Int. J. Control, vol. 74, no. 5, pp. 432-444, 2001.

[8] — "Experimental application of direct adaptive control laws for adaptive stabilization and command following," in Proc. Conf. Decision Control, Phoenix, AZ, Dec. 1999, pp. 779-783.

[9] H. K. Khalil, Nonlinear Systems, 2nd ed. Upper Saddle River, NJ: Prentice-Hall, 1996. 